\title{
Image Processing-based Automatic Pupillometry on Infrared Videos
}

\author{
György Kalmár, Alexandra Büki, Gabriella Kékesi, \\ Gyöngyi Horváth, and László G. Nyúl ${ }^{a}$
}

\begin{abstract}
Pupillometry is a non-invasive technique that can be used to objectively characterize pathophysiological changes involving the pupillary reflex. It is essentially the measurement of the pupil diameter over time. Here, specially designed computer algorithms provide fast, reliable and reproducible solutions for the analysis. These methods use a priori information about the shape and color of the pupil. Our study focuses on measuring the diameter and dynamics of the pupils of rats with schizophrenia using videos recorded with a modified digital camera under infrared (IR) illumination. We developed a novel, robust method that measures the size of a pupil even under poor circumstances (noise, blur, reflections and occlusions). We compare our results with measurements obtained using manual annotation.
\end{abstract}

Keywords: pupillometry, ray propagation, energy attenuation

\section{Introduction}

In medical diagnostics, non-invasive techniques and their applications are actively studied today. Most of the well-known ones rely on computer aided imaging, such as fMRI, CT, SPECT, and PET. One of the simpler techniques is pupillometry, which can be used to analyze diseases and their pathophysiological changes involving the pupillary reflex. Essentially, it is the measurement of the diameter of a pupil during light stimuli, which induce pupillary light reflex, i.e. the contraction of a pupil in response to light. Pupillary light reflex-derived metrics can be used to monitor the extent of neurological diseases and their response to therapy. Earlier studies examined the parasympathetic function within the context of schizophrenia through the use of pupillometry. These investigations demonstrated that pupil diameter is larger and the reflex is slower in patients with schizophrenia. The

\footnotetext{
${ }^{a}$ Department of Image Processing and Computer Graphics, University of Szeged, E-mail: \{kalmargy, nyul\}@inf.u-szeged.hu

${ }^{b}$ Department of Physiology, University of Szeged, E-mail: \{buki.alexandra, kekesi.gabriella, horvath.gyorgyi\}@med.u-szeged.hu
} 


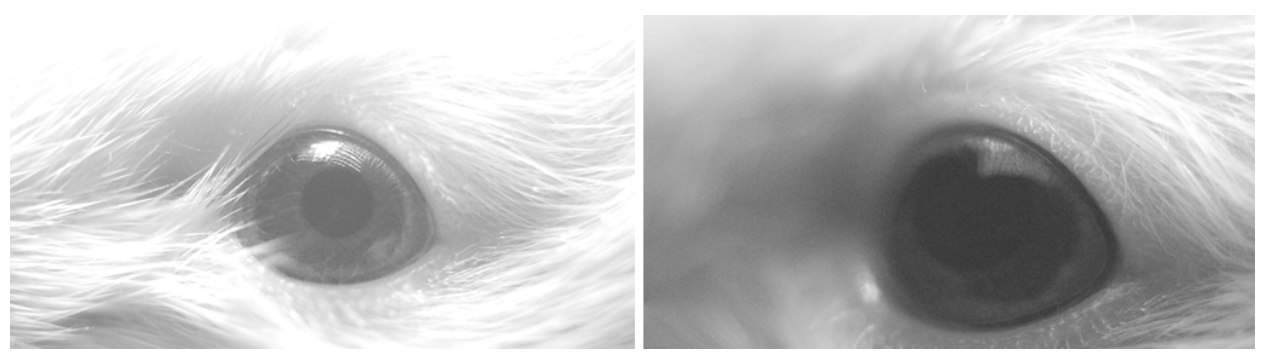

Figure 1: Example for a good (left) and a bad (right) quality image.

basic idea of pupillometry is to record the pupillary reflex with a camera and after the controlled experiments, the video recordings are processed. Measuring the diameters manually frame-by-frame with the aid of a simple program is time consuming and non-reproducible in cases where the number of subjects is high. Automated, specially designed computer algorithms provide faster, more reliable and reproducible solutions for the analysis. These methods use a priori information about the darkness and circular shape of the pupil. From an image processing perspective, the problem is essentially that of circle detection. A combination of well-known circle detection methods with special, pupillometry-related information should result in accurate and robust solutions.

Our study focuses on measuring the diameter of rats' pupils using infrared (IR) videos recorded by a digital camera with an IR filter. Medical research supported by our efforts investigates the influence of schizophrenia on pupillary light reflex. During the experiments, rats are held by hand on a desk while a short light impulse is shone into their eyes. The camera with a fixed focal length is properly positioned prior to the experiments to be able to record the response of pupil to the light stimulus. The videos, however, have certain quality defects. Breathing and slight movements of the rats cause scattering movements and significant blur, which makes the segmentation of pupil impossible with simple binarization. Beyond these artifacts, the rats' eyes have a red color, without any melanocyte, resulting in a low contrast between the pupil and the iris. Low lighting conditions force a higher ISO level value during the recordings, and this leads to noisier images. The reflections of the illuminating IR LED source obscure a large part of the pupil boundary, while other overlying entities such as the whiskers of the animal can make the pupil virtually undetectable. In Figure 1 two sample images for a good and a bad quality video are shown. On the left hand side the dark region in the middle of the eye is the pupil region, which is easy-to-detect, in contrast to the picture on the right hand side; the image is blurred, has a low contrast and the pupil region is barely distinguishable from the iris region.

To overcome the above-mentioned difficulties and reliably, accurately detect the pupil, and automatically measure its parameters, we developed a novel ray propagation-based method with energy attenuation. It can find the fine contrast changes at the boundary of the pupil, while tolerating noise, reflections, occlusions 
and give a good result even with blurred images. The proposed technique can measure the diameter of a pupil with a small size and sharp edge and also big, blurred, noisy ones using the same procedure.

The paper is organized as follows. In Section 2, related works are mentioned, then in Section 3 we present our proposed automated procedure. In Section 4, we discuss in detail the functionality of the energy-based technique. Then, in Section 5, we elaborate on our estimation and measuring procedure. This is followed by our evaluation and presentation of results in Section 6. Lastly, in Section 7, we summarize our findings and make suggestions for future study.

\section{Related works}

In image processing, there are many well-known sophisticated circle detection algorithms based on various concepts. One of the most popular approaches is based on the Hough transform. The Circle Hough Transform (CHT) detects circles with a given radius. It uses a 3 -D parameter space and local maxima searching in a so-called accumulator space. The input for the CHT is an edge image. For each edge point, it increments the accumulator values that correspond to points located at a given distance from the selected edge point. The accumulator space is a $3-\mathrm{D}$ space. Two dimensions correspond to the coordinates of the center of the circles, and the third to the radius. After processing all edge points, local maxima appear at the accumulator points that correspond to the circle centers. Modifications to the CHT (use of edge orientation, use of a single accumulator space for multiple radii, use of phase to code radii, randomization, etc.) were introduced to reduce the computational cost and improve the detection rate. For more details see $[2,10,17]$. We cannot apply a CHT directly because there may be a substantial blur, part of the pupil boundary may not be observable in the edge image, and the pupil shape may not be a perfect circle.

Another idea is based on the fact that the image gradients at the circle boundary point outwards from the center of the circle (dark circle, white background). In [14], a fast circle detection algorithm is described. First, the gradient image is calculated and because of the symmetry of the circle, for each vector there will be a pair of vectors in opposite directions. For a given vector $\mathbf{V}$, its pair vector W satisfies two basic conditions; namely the absolute difference between the two directions should be nearly 180 degrees and the angle between the vector $\mathbf{V}$ and the line connecting the bases of vectors $\mathbf{V}$ and $\mathbf{W}$ should be nearly 0 degrees. In the next phase, the corresponding vector pairs are formed. Then, candidate circles are computed for all vector pairs. Afterwards, suitable circles are extracted from the candidates (accumulator matrix, clustering). In our case, the reflections and blurry boundary do not generate a consistent gradient vector space to be able to detect correctly the circular shaped pupil.

In recent years, optimization-based methods have become quite popular. These use well-known optimization techniques to find local extrema in an appropriately chosen function space, some of them having been inspired by biological notions. 
Such approaches include the Genetic Algorithms (GA) [1], Bacterial Foraging Algorithm Optimizer (BFAO) [8], Harmony Search Optimization [4], Artificial Bee Colony (ABC) optimization [6], and several others [5, 7].

Pupillometry is a long-known method that is used to objectively characterize the pupil's response to stimuli. Eye tracking, pupil center detecting, and measuring th pupil diameter form parts of an automated pupillometry process. Simpler, earlier methods try to determine a suitable threshold value and binarize the eye image, which is processed by shape analysis or ellipse fitting algorithms [3, 11, 12, 13, 15]. In [9], the authors propose a fully automated procedure for pupil segmentation based on the level set theory. The level set formulation can handle the complex topology of biomedical images regardless of the initial level set configuration. They used a 4-level segmentation, that is suitable for extracting the pupil from the eye picture and measure various morphological parameters, such as the pupil's diameter, centroid and area. The authors of [18] developed an algorithm that utilizes the curvature characteristics of the pupil boundary to determine the visible portion of the pupil, which provides improved estimates of the pupil center when it is obscured by a host of artifacts. The curvature algorithm discriminates between edge points that lie on the smooth pupil boundary and those that lie on the intersection of the pupil with eyelids, eyelashes, corneal reflections or shadows. The non-occluded boundary points are used as input to an ellipse-fitting procedure that offers a robust estimate of the pupil center. The above-mentioned papers describe acceptable solutions for the pupil detection problem, but these methods have their shortcomings when it comes to handling reflections, blur and low contrast difference.

\section{Pipeline of the automated pupillometry}

Our procedure seeks to support medical staff in their work. In these studies on the connection between schizophrenia and pupillary reflex, they need to analyze sets of videos. Processing hundreds of videos by hand is time consuming and nonreproducible. To overcome this problem, we developed an automated system that can bring a significant speed-up in the processing stage. The input of this system is a video, which contains the recorded pupillary response to a single stimulus. The output is a diagram, that expresses a change in the pupil diameter over time. The pipeline of the proposed automated process is shown in Figure 2.

\subsection{Movement filtering}

As we mentioned above, the test subjects breathe and make small movements because they have only been slightly sedated and are held by hand. This causes scattering movements of the eye in the videos. To compensate for this artifact, we stabilize the recordings with the help of the Kanade-Lucas-Tomasi (KLT) point tracker [16]. We assume that the size and shape of the eye do not change (rats do not blink during the recordings), and only translational transformations occur between the consecutive frames. The KLT algorithm tracks corner points throughout the 


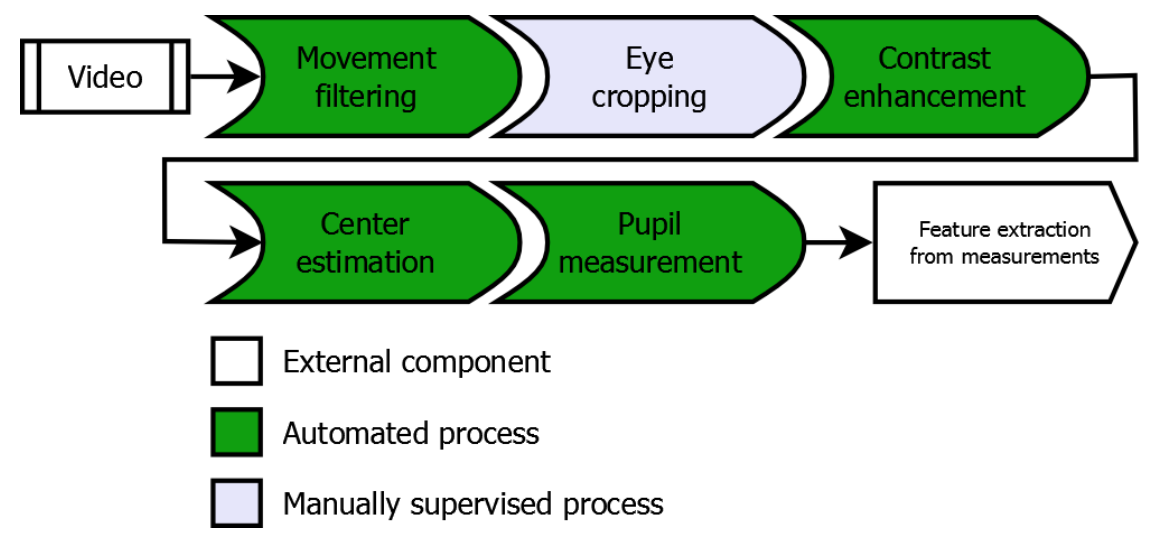

Figure 2: Pipeline of the automated pupillometry.

video. And in each frame the successfully tracked points and their initial positions are connected by translational vectors. The median value of translations is then used to translate the whole frame back to the assumed initial position. The result of this stage is a video, where the eye of the rat has been stabilized.

\subsection{Eye cropping and contrast enhancement}

The region of interest (ROI) in our case is, of course, the eye region. The diameter of the pupil is expressed relative to the size of the iris. The boundary of the eye is not defined exactly because of shadows and hairs. Experience is necessary to select the appropriate region that just contains the eye. We generate an initial guess based on the projections of dark pixels to the horizontal and vertical axes, but supervision is required. We developed a simple graphical user interface to assist the selection of the ROI. After defining a bounding box that contains the eye, we crop that area from each frame, because we assume that after the stabilization process the eye's position will not change. This in turn reduces the amount of pixel data that will be processed.

One of the major problems is that the contrast between the pupil and iris is very low. The contrast enhancement process attempts to increase the difference by assuming that the minimal pixel intensity within the eye region corresponds to the pupil region. After inspecting the intensity histogram of the eye area, it is clear that it is bimodal. Using this information we can calculate an optimal threshold value that separates the iris region and pupil region. The minimal pixel intensity and the computed threshold value give the two main points for a histogram stretching algorithm. 


\subsection{Center point estimation}

In the next phase, we need to get a good estimation of the pupils's center point. In this phase, the entire video is processed and the output is a position (in two coordinates) that most likely corresponds to the center of the pupil region. This process heavily relies on our novel ray-propagation-with-energy-attenuation technique, which is described below. The center point estimation will be discussed in more details in Section 5 .

\subsection{Pupil measurement}

Lastly, the diameter of the pupil is determined for each frame. We fit an ellipse on the filtered boundary points produced by the novel energy attenuation model-based ray propagation method. The output of the process is a diagram which expresses the change of pupil's parameters during the experiments.

\section{Ray propagation with energy attenuation}

In the IR videos the contrast between the pupil and iris region is low. One of the main reasons is that the rats' eye contains no melanocyte. Another is that the recordings are made with a modified digital camera with IR filter which requires the use of an intense external IR light source. The relative position between the rat and light source is not constant, and this affects the amount of light reflected from the iris. These factors lead to a significant variation in the video quality and make the proper pupil detection challenging. The proposed method can handle the dynamic change of intensity levels, not only among videos, but also frame-by-frame; and it remains robust to noise.

In the following, we shall assume that the pupil region has a darker color (lower intensity) than the surrounding iris region and that the video frames contain only one dark circular region which is enclosed by a brighter region. It does not matter that only a part of the pupil may be visible or it is affected by significant amount of noise and blurring. We also assume that the center of pupil has been accurately estimated and that it remains the same during the recordings.

The proposed method uses notions and ideas taken from the physics of wave (ray) propagation. The rays have an initial energy which is gradually absorbed by the medium during the propagation because of scattering. The amount of energy loss is proportional to the attenuation coefficient of the material. A medium with no attenuation is called a vacuum. Relating to these principles, the idea is to cast rays from a point and use pixel intensities as measures of attenuation capabilities, and trace them while traveling through the image, then use the information of energy loss characteristics to learn more about the structure of surrounding regions. Higher pixel intensities mean a higher attenuation.

Rays with initial energies radiate uniformly from the estimated pupil center. We trace them until their energy is completely absorbed and record the position of the endpoint $\varepsilon$, where it occurred. Because of the above-mentioned notions are 
inherently related to direction and distance from a center point, positions will be determined in the polar coordinate system, whose origin is the estimated center point of the pupil and the polar axis is drawn horizontal and pointing to the right. We will denote the angular and the radial coordinates by $\theta$ and $r$, respectively. At a point $[\theta, r]$, the pixel intensity is $I_{\theta r}$. For a ray with direction $\theta$, the radial coordinate of its endpoint will be denoted by $\varepsilon_{\theta}$.

The basic idea is to treat the pupil region as a vacuum. For each frame, we calculate the vacuum intensity

$$
I_{v}=\frac{1}{|R|} \sum_{x, y \in R} I_{x y} .
$$

Here, $I_{v}$ is defined as the average pixel intensity of a small region $R$ around the estimated pupil center. We shift the original pixel intensities $I$ to $I^{*}$ as follows: $I^{*}=\max \left[I-I_{v}, 0\right]$. If a ray with energy $E$ travels through a pixel at $[\theta, r]$ with intensity $I_{\theta r}^{*}$, we can update the current ray energy using

$$
E:=E-f\left(I_{\theta r}^{*}\right) .
$$

We will use a quadratic function for $f$ to emphasize the contrast between the pupil and iris.

Let us define the set of initial energies $E_{I}=\left\{e_{k}=\alpha+k \delta \mid k \in \mathbb{N}\right\}$, where $\alpha$ is an initial offset energy and $\delta$ is the step size between the different energy levels. It is worth noting that a reasonable upper limit for the energy levels is definable by considering the intensity values in the image. One possibility might be the determination of the minimal amount of energy required to reach the border of the selected ROI.

The higher the initial energy the more a ray can penetrate the high intensity region. If the region of the pupil is noise-free, rays with low initial energy reach the border of the pupil and stop at or close to the boundary points. If there is noise, some of the low energy rays stop before reaching the boundary, but most of the higher energy rays stop at the boundary.

For a given direction $\theta$, for each initial energy $e_{i} \in E_{I}$ we can calculate endpoint $\varepsilon_{\theta}^{i}$ of the corresponding rays. The set of endpoints in a given direction $\theta$ will be denoted by $S_{\theta}=\left\{\varepsilon_{\theta}^{i}\right\}_{i=1, \ldots, n}$, where $n$ is the number of rays that have been cast (the number of distinct initial energy levels). The propagation of rays in a homogeneous medium with a fixed linear step size difference in their initial energies leads to linearly spaced endpoints. In our case the structure of the eye is inhomogeneous, i.e. the pupil region's intensity is close to that of the vacuum, unlike the outer regions that mostly have higher attenuation. This fact causes a clustering in the endpoint positions. Overall, casting rays with monotonically growing initial energy leads to clusterings near the boundary points - specifically, near points with higher intensities. The main challenge is to properly select the correct clustering region that corresponds to the pupil boundary. We use hierarchical clustering to form the clusters. All the endpoints in a certain direction $\theta$ that are at a very small distance 
from each other belong to the same cluster.

$$
c_{\theta j}=\left\{\varepsilon_{\theta}^{i} \mid \text { maximal shortest distance between endpoints }<d\right\},
$$

where $d$ is a given small constant, $c_{\theta j}$ is a cluster of endpoints (where the distance between two properly selected endpoints is less than $d$ ). By using the initial assumption that beyond the pupil boundary there are mostly higher intensities, it is not hard to see that the rays with growing initial energy belong to the same cluster after reaching the pupil's border, because they can only penetrate a little bit into the higher intensity region, and their stop positions will be close to each other. Let $C_{\theta}=\left\{c_{\theta j}\right\}_{j=1, \ldots, m}$ denote the set of clusters for the direction $\theta$, where $m$ is the number of clusters. As $C_{\theta}$ is the clustered version of $S_{\theta}, \bigcup_{j=1}^{m} c_{\theta j}=S_{\theta}$.

By using a set of initial energies with a higher density (i.e. smaller $\delta$ ), it is easy to show that the cluster corresponding to the boundary region has the maximal cardinality.

Now let us find the cluster $c_{\theta k}$, where $k$ is

$$
k=\underset{j}{\arg \max }\left|c_{\theta j}\right|,
$$

and $|c|$ is the cardinality of cluster $c$. In the selected cluster, the closest endpoint to the estimated pupil center (i.e. the endpoint with the smallest radial coordinate $r$ ) corresponds to the pupil boundary point $\beta_{\theta}=\left[\theta, r_{\text {min }}\right]$, where:

$$
r_{\text {min }}=\min _{i}\left\|\varepsilon_{\theta}^{i}\right\|, \varepsilon_{\theta}^{i} \in c_{\theta k} .
$$

For all directions parameterized by $\theta$, collect the selected border points into a set denoted by $\mathbf{B}=\left\{\beta_{\theta}\right\}_{\theta}$, where $\theta$ is a set of directions; in our case $\theta=\{1,2, \ldots, 359,360\}$. This $\mathbf{B}$ is the output of our method, which is used later on to estimate the size of the pupil.

Figure 3 provides an illustration of he above-described process. The original image in Figure 3(a) has a good contrast but is noisy and blurry. For better visualization, only the pupil area is shown. We can follow the above-mentioned steps in a given direction. In Figure 3(d), the energy curves of rays with various initial energies are shown, and the square root of values are have been plotted to highlight lower initial energies. The curves are colored according to their endpoint cluster membership, which is shown in Figure 3(e). In Figure 3(g), the selected endpoints for all directions are indicated. Then in Figure 3(h), the result of filtering and ellipse fitting is shown. This last step will be discussed next.

\section{Center point estimation and diameter measur- ing}

Earlier, it was assumed that we have a good estimate for the pupil center point. In our approach, we use a geometrical relation between the center of circle and 


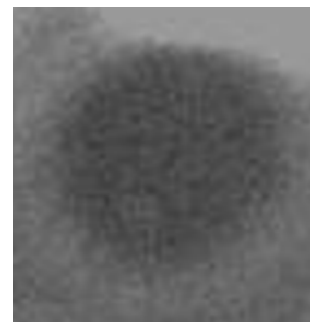

a)

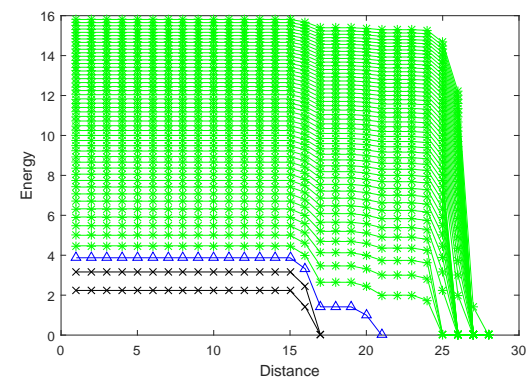

d)

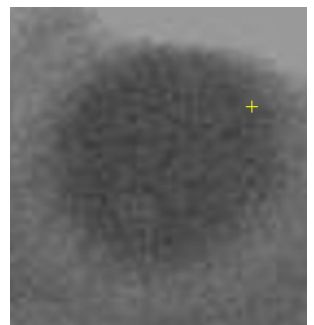

f)

b)

g)
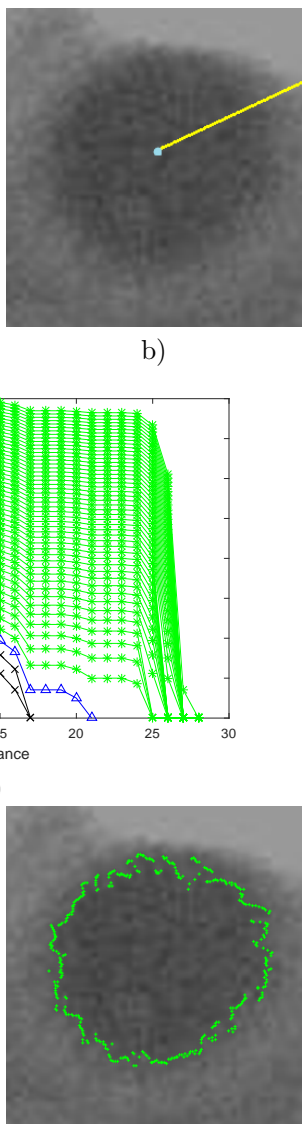

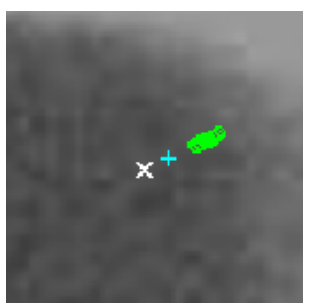

e)

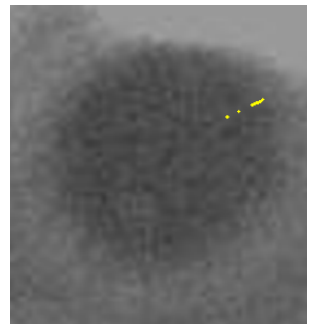

c)

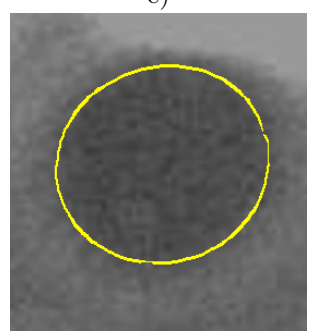

h)

Figure 3: a) Original image, pupil area, b) selected direction of ray propagation, c) endpoints of rays, d) chart of ray energy curves at different initial energy levels, e) clustered endpoints, f) selected endpoint in the given direction, g) detected endpoints for all directions, h) the result of filtering and ellipse fitting.

its chords. We pick a point at random within the circle (Figure 4(a)) and draw chords through this point (Figure 4(b)). The perpendicular bisectors of the chords intersect at one single position, which is the center of the circle (Figure 4(c)).

The estimation process starts by placing seed points located in a grid. For each seed point having a pixel intensity near the minimal intensity in the image, we do the following. By using the energy attenuation model-based ray propagation method (Section 4), we cast rays from the actual seed point and find the most probable boundary points which give the endpoints of chords. After filtering the chords by their length, we calculate the perpendicular bisectors. It should be added that because of the inaccuracy of chord determination, the calculated lines 


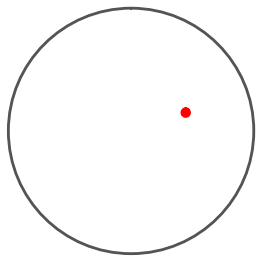

a)

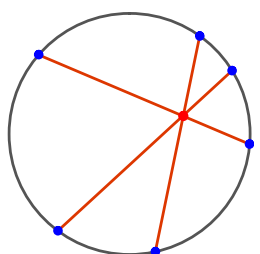

b)

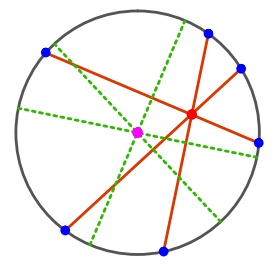

c)

Figure 4: Circle center point estimation: a) random point within a circle, b) chords through the selected point, c) perpendicular bisectors and the center point of circle.

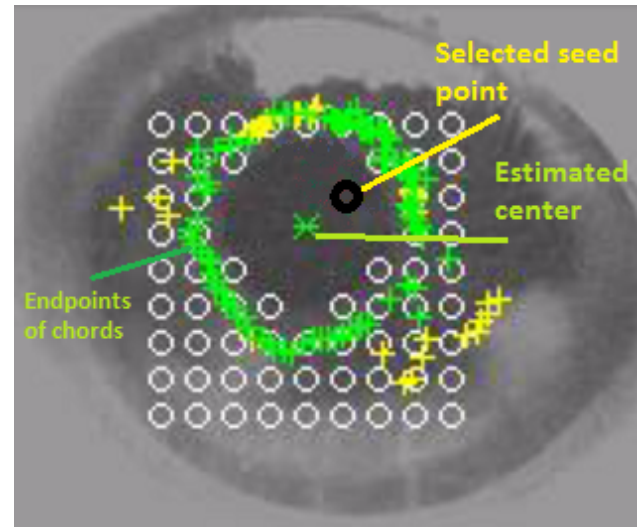

Figure 5: Estimation of the pupil center point.

do not intersect at a single point. To overcome this problem, we do a Least-Squares estimation to find the best intersection point for the given lines. Distances between the estimated center point and previously found boundary points are computed. The standard deviations of these distances then serve as scores for the corresponding estimated positions. After processing all the frames from the video, we have a huge number of pairs containing the estimated positions and its score. The output is the point that has the minimal score value. In Figure 5, the estimation process is shown. The seed points marked by white circles are filtered out at the beginning because their intensity was too high. The yellow plus signs mark the endpoints that have been filtered out because the corresponding chord length was too high compared to the median length of the others. It can be seen in the figure that from the selected seed point (black circle), the method estimated the center point (marked by a green star) with an acceptable accuracy.

After the selection of the best estimated center point, each frame is processed using this point as the source of radiation. The output for each frame is a set of endpoints and their distances, which may contain faulty detections due to high intensity reflections, undetectable boundary sections and occlusions. These factors 
occur randomly compared to the regularity of pupil boundary points. Using this assumption, we will quantize the distances of endpoints and calculate their histogram. From the histogram, the distance with maximal magnitude is chosen since it is the most probable approximation of the pupil radius. Using this value, endpoint filtering is performed. Only endpoints with a distance close to the approximated radius are kept, on which Least-Squares based ellipse fitting is performed. Due to the relative position between the animal and the camera, the pupil is not necessarily a perfect circle; it may have a slightly elliptical shape. Anatomically the pupil is a circle whose radius is shortened in one direction in the video images due to the viewing angle. To produce the most realistic measure of the pupil diameter, we extract the longest axis from the fitted ellipse. If there is insufficient information (too few endpoints after filtering) to fit an ellipse, we just use the approximated radius length. The output of the automated process is the curve of the pupil's diameter change during the experiment, which is median filtered to eliminate bad measurements. Sample results of filtering, measurement and ellipse fitting phase are shown in Figure 6. In each picture, the green symbols represent the retained endpoints, while the red ones denote the outfiltered endpoints. The ellipses fitted to the retained endpoints are represented by yellow colored ellipses.

\section{$6 \quad$ Evaluation and results}

We evaluated our automated pupillometry on 20 videos, each containing 450 frames. The outcome of the automated process was then compared to results obtained by manual correction. We also developed a graphical user interface to support the correction of inaccuracies. After every fifth frame the user can correct the diameter length by hand if necessary. We did it this way, because the spectra of the intensity parameters is bigger than those having the same number of supervised measurements in consecutive frames. It should be added that in most cases the pupil boundary is not sufficiently sharp to place separating points exactly on it. Acceptable measurement means that a human would place the boundary point very close (within a few pixels) to the point representing the automatically computed position. We compared our result only for the 1800 supervised frames and we list the results of statistical analysis in Table 1 . For each frame $f \in F_{v}$ in video $v \in V$, the relative percentage error (RPE) of the diameter measurement is

$$
R P E_{v f}=100 \% \cdot \frac{\left|d_{v f}-\hat{d}_{v f}\right|}{\left|d_{v f}\right|},
$$

where $d_{v f}$ is the corrected diameter, $\hat{d}_{v f}$ is the estimated pupil diameter, $F_{v}$ is the set of indices of supervised frames in video $v \in V, V=\{1,2, \ldots, 20\}$. In Table 1, the rows correspond to the aggregation of RPE for all frames in a video, the columns correspond to the aggregation of the per video performances for each video in the test dataset. For instance, for video $v$, the mean relative percentage error $\mu R P E$ 

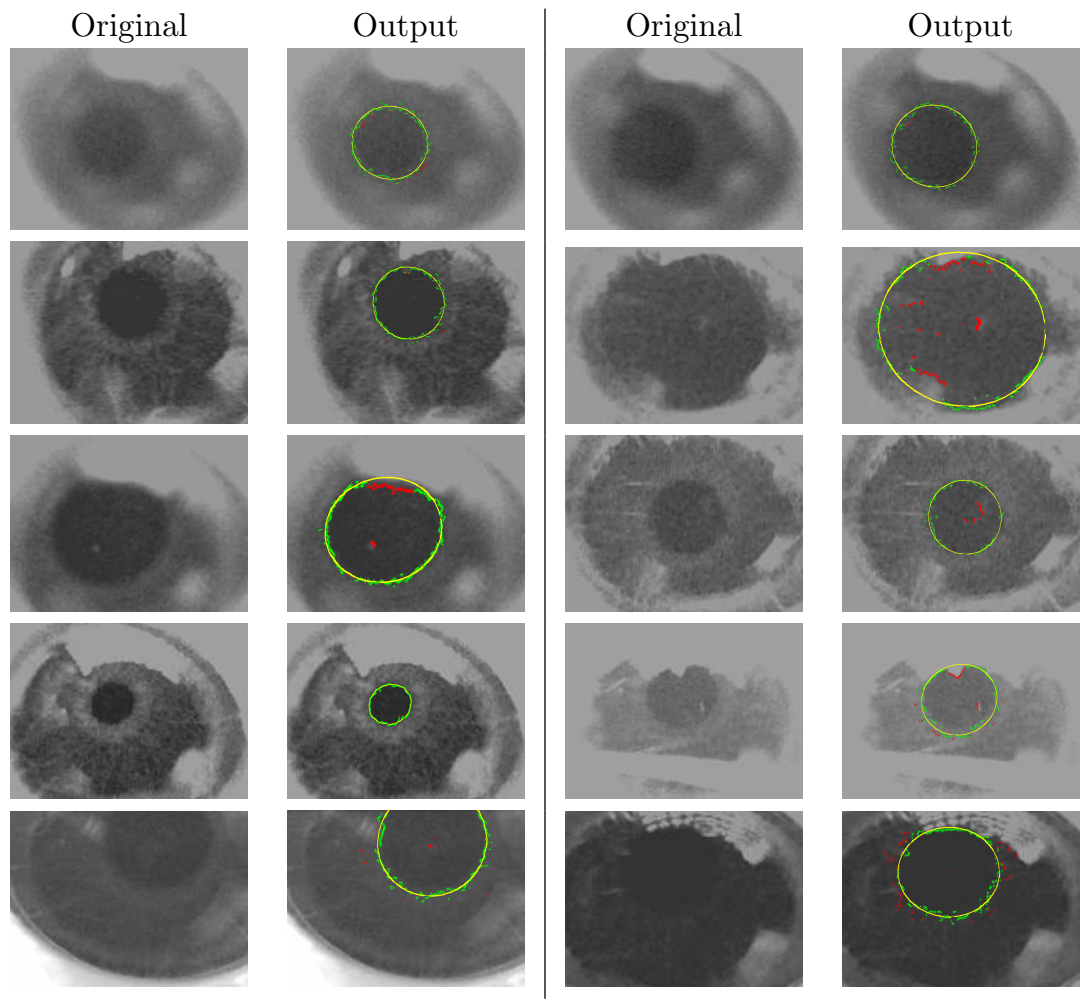

Figure 6: Results of endpoint filtering and ellipse fitting. The green symbols denote the retained endpoints, and the red ones denote the outfiltered endpoints. The yellow colored ellipses are fitted on the retained endpoints.

is just

$$
\mu R P E=\frac{1}{\left|F_{v}\right|} \sum_{f \in F_{v}} R P E_{v f},
$$

where $\left|F_{v}\right|$ is the number of supervised frames in video $v$. The maximum of the $\mu R P E$ values in our case is $4.85 \%$ (which appears in the second data cell in the second row of Table 1). The upper part of Table 1 shows the results of analysis for all supervised frames, while the bottom part shows the same but only for the corrected frames. The last row gives information about the ratio of corrected frames.

As can be seen, for each supervised frame the mean relative percentage error is less than $2 \%$, and in the corrected frames is around $4 \%$, which is sufficiently accurate to assist medical staff in their work. On average, $40 \%$ of the frames were corrected by hand during supervision, but in most cases there were only minor differences between the estimated length and real diameter length. The proposed automatic method processes 3 frames per second, which includes ray tracking, filtering, hierarchical clustering, ellipse fitting and output video rendering. 
Table 1: Performance analysis of the proposed method. The rows correspond to the aggregation of RPE for all frames in a video, while the columns correspond to the aggregation of the per video performances for each video in the test dataset.

\begin{tabular}{|c|c|c|c|c|c|c|}
\hline & \multirow{3}{*}{$\begin{array}{c}\text { Minimum } \\
0.48\end{array}$} & \multirow{3}{*}{$\begin{array}{c}\text { Maximum } \\
22.41\end{array}$} & \multirow{3}{*}{$\begin{array}{l}\text { Mean } \\
10.64\end{array}$} & \multirow{3}{*}{$\begin{array}{c}\begin{array}{c}\text { Standard } \\
\text { deviation }\end{array} \\
5.32\end{array}$} & \multirow{3}{*}{$\begin{array}{c}\text { Median } \\
8.79\end{array}$} \\
\hline & & & & & & \\
\hline \multirow{4}{*}{$\begin{array}{l}\text { All } \\
\text { supervised } \\
\text { frames }\end{array}$} & $\begin{array}{l}\text { Maximum relative } \\
\text { error (\%) }\end{array}$ & & & & & \\
\hline & $\begin{array}{c}\text { Mean } \\
\text { relative error (\%) }\end{array}$ & 0.60 & 4.85 & 1.95 & 1.38 & 1.53 \\
\hline & $\begin{array}{c}\text { Standard } \\
\text { deviation of relative } \\
\text { error }(\%)\end{array}$ & 0.95 & 6.79 & 2.68 & 1.35 & 2.44 \\
\hline & $\begin{array}{c}\text { Median } \\
\text { of relative error (\%) }\end{array}$ & 0.00 & 3.10 & 0.90 & 1.23 & 0.00 \\
\hline \multirow{4}{*}{$\begin{array}{l}\text { Corrected } \\
\text { frames }\end{array}$} & $\begin{array}{l}\text { Mean } \\
\text { relative error (\%) }\end{array}$ & 1.41 & 8.52 & 4.13 & 1.63 & 4.13 \\
\hline & $\begin{array}{c}\text { Standard } \\
\text { deviation of relative } \\
\text { error }(\%)\end{array}$ & 0.98 & 7.19 & 2.94 & 1.58 & 2.46 \\
\hline & $\begin{array}{c}\text { Median } \\
\text { of relative error (\%) }\end{array}$ & 1.02 & 7.32 & 3.53 & 1.63 & 3.30 \\
\hline & $\begin{array}{l}\text { Corrected } \\
\text { frames }(\%)\end{array}$ & 15.28 & 88.89 & 46.38 & 23.03 & 40.97 \\
\hline
\end{tabular}

Processing an input video takes around 3 minutes.

\section{Conclusions}

Here, we presented and described an automated process for measuring the pupil diameter in an infrared video. To accurately detect the boundary of the pupil, a novel energy attenuation-based ray propagation method was introduced. It can handle the wide spectra of intensity parameters, along with low contrast, blur and occlusions. The estimation of the pupil center point and robust diameter measuring was also outlined. We evaluated the proposed process on 20 videos and achieved an overall error rate below $2 \%$. In the future, we would like to parallelize our computations and use more robust filtering of real boundary points. Also, it would be good to objectively compare our results with those produced by other similar methods. 


\section{References}

[1] Ayala-Ramirez, Victor, Garcia-Capulin, Carlos H., Perez-Garcia, Arturo, and Sanchez-Yanez, Raul E. Circle detection on images using genetic algorithms. Pattern Recognition Letters, 27(6):652-657, 2006.

[2] Chen, Teh-Chuan and Chung, Kuo-Liang. An efficient randomized algorithm for detecting circles. Computer Vision and Image Understanding, 83(2):172191, 2001.

[3] Cho, J.M., Lee, S.J., Kim, J.K., Choi, H.H., Kwon, O.S., and Kwon, J.W. A pupil center detection algorithms for partially-covered eye image. In TENCON 2004. 2004 IEEE Region 10 Conference, volume A, pages 183-186. IEEE, 2004.

[4] Cuevas, Erik, Ortega-Sánchez, Noé, Zaldivar, Daniel, and Pérez-Cisneros, Marco. Circle detection by harmony search optimization. Journal of Intelligent $\&$ Robotic Systems, 66(3):359-376, 2012.

[5] Cuevas, Erik, Osuna-Enciso, Valentn, Wario, Fernando, Zaldvar, Daniel, and Prez-Cisneros, Marco. Automatic multiple circle detection based on artificial immune systems. Expert Systems with Applications, 39(1):713-722, 2012.

[6] Cuevas, Erik, Sención-Echauri, Felipe, Zaldivar, Daniel, and Pérez-Cisneros, Marco. Multi-circle detection on images using artificial bee colony (ABC) optimization. Soft Computing, 16(2):281-296, 2012.

[7] Cuevas, Erik, Zaldivar, Daniel, Pérez-Cisneros, Marco, and Ramírez-Ortegón, Marte. Circle detection using discrete differential evolution optimization. Pattern Analysis and Applications, 14(1):93-107, 2011.

[8] Dasgupta, Sambarta, Das, Swagatam, Biswas, Arijit, and Abraham, Ajith. Automatic circle detection on digital images with an adaptive bacterial foraging algorithm. Soft Computing, 14(11):1151-1164, 2010.

[9] De Santis, A. and Iacoviello, D. Optimal segmentation of pupillometric images for estimating pupil shape parameters. Computer Methods and Programs in Biomedicine, 84(2-3):174-187, 2006.

[10] Illingworth, J. and Kittler, J. A survey of the Hough transform. Computer Vision, Graphics, and Image Processing, 44(1):87-116, 1988.

[11] Kim, Jieun and Park, Kyungmo. An image processing method for improved pupil size estimation accuracy. In Engineering in Medicine and Biology Society, 2003. Proceedings of the 25th Annual International Conference of the IEEE, volume 1, pages 720-723. IEEE, 2003.

[12] Lee, J.C., Kim, J.E., Park, K.M., and G., Khang. Evaluation of the methods for pupil size estimation: On the perspective of autonomic activity. In Engineering in Medicine and Biology Society, 2004. IEMBS '04. 26th Annual International Conference of the IEEE, volume 2, pages 1501-1504. IEEE, 2004. 
[13] Miller, D.B., Benton, B.J., Hulet, S.W., Mioduszewski, R.J., Whalley, C.E., Carpin, J.C., and Thomson, S.A. An image analysis method for quantifying elliptical and partially obstructed pupil areas in response to chemical agent vapor exposure. In Bioengineering Conference, 2003 IEEE 29th Annual, Proceedings of, pages 63-64. IEEE, 2003.

[14] Rad, Ali Ajdari, Faez, Karim, and Qaragozlou, Navid. Fast circle detection using gradient pair vectors. In Proc. VIIth Digital Image Computing: Techniques and Applications, pages 879-888, 2003.

[15] Ritter, Nicola, Cooper, James, Owens, Robyn, and Van Saarloos, Paul P. Location of the pupil-iris border in slit-lamp images of the cornea. In Image Analysis and Processing, 1999. Proceedings. International Conference on, pages 740-745. IEEE, 1999.

[16] Tomasi, Carlo and Kanade, Takeo. Detection and tracking of point features. School of Computer Science, Carnegie Mellon Univ. Pittsburgh, 1991.

[17] Yuen, HK, Princen, J, Illingworth, J, and Kittler, J. Comparative study of Hough transform methods for circle finding. Image and Vision Computing, 8(1):71-77, 1990.

[18] Zhu, Danjie, Moore, Steven T., and Raphan, Theodore. Robust pupil center detection using a curvature algorithm. Computer Methods and Programs in Biomedicine, 59(3):145-157, 1999. 